\title{
Conservation laws, soliton-like and stability analysis for the time fractional dispersive long-wave equation
}

\author{
Abdullahi Yusuf ${ }^{1,2^{*}}$ (D, Mustafa Inc ${ }^{1}$, Aliyu Isa Aliyu ${ }^{1,2}$ and Dumitru Baleanu 3,4
}

\section{"Correspondence:}

yusufabdullahi@fud.edu.ng

1 Department of Mathematics,

Science Faculty, Firat University,

Elazig, Turkey

${ }^{2}$ Department of Mathematics,

Science Faculty, Federal University

Dutse, Jigawa, Nigeria

Full list of author information is

available at the end of the article

\begin{abstract}
In this manuscript we investigate the time fractional dispersive long wave equation (DLWE) and its corresponding integer order DLWE. The symmetry properties and reductions are derived. We construct the conservation laws (Cls) with

Riemann-Liouville (RL) for the time fractional DLWE via a new conservation theorem. The conformable derivative is employed to establish soliton-like solutions for the governing equation by using the generalized projective method (GPM). Moreover, the Cls via the multiplier technique and the stability analysis via the concept of linear stability analysis for the integer order DLWE are established. Some graphical features are presented to explain the physical mechanism of the solutions.
\end{abstract}

Keywords: Time fractional PDEs; RL fractional derivative; Cls; Solitons; Stability analysis

\section{Introduction}

Fractional calculus has mesmerizing features due to its pragmatic applications in various areas of science, social science, finance, and engineering to mention a few. Owing to this, a lot of meaningful definitions that have to do with fractional derivatives have been proposed by different authors in order to fully explain the memory effect [1-4]. Among the existing derivatives, we mention Grunwald-Letnikov, Marchaud, Riemann-Liouville, Hadamard, modified Riemann-Liouville, and Caputo [5-10].

Recently, a new definition of derivative has been introduced, and it is called the conformable derivative. The newly introduced conformable derivative satisfies a lot of characteristics such as product and quotient formulas, and it is used to model some physical problems [11]. Several authors have utilized this definition in the real world problems [12-16].

Cls have originated from the pragmatic phenomena such as energy, mass, and momentum [17]. The Cls have been utilized for developing numerical techniques, proving the existence and uniqueness of solutions [18], analysis of the internal characteristics like recursion operators, bi-Hamiltonian structures [19]. It should be noted that there have been numerous generalizations of Noether's theorem and Euler-Lagrange's [20] associating to several definitions of fractional derivative to establish Cls for fractional nonlinear PDEs possessing fractional Lagrangians [21-23]. Furthermore, nonlinear physical phenomena

(c) The Author(s) 2018. This article is distributed under the terms of the Creative Commons Attribution 4.0 International License (http://creativecommons.org/licenses/by/4.0/), which permits unrestricted use, distribution, and reproduction in any medium, provided you give appropriate credit to the original author(s) and the source, provide a link to the Creative Commons license, and indicate if changes were made. 
may be explained through establishing exact solutions. This has brought a strong motivation to authors to obtain exact solutions using different schemes [24-51].

In the present paper, we investigate the Cls and soliton-like solutions of the time fractional DLWS with RL and conformable derivatives, respectively. Moreover, we compute the Cls via the multiplier technique and the stability analysis via the concept of linear stability of the integer order DLWS.

\section{Basic tools}

In this section, some preliminaries pertaining to symmetry analysis for time fractional PDEs will be highlighted. Let us assume that we have a system of time fractional PDEs given as

$$
\begin{gathered}
\frac{\partial^{\alpha} U}{\partial T}=F\left(X, T, U, V, U_{X X}, U_{X X X}, \ldots\right), \\
\frac{\partial^{\alpha} V}{\partial T}=F\left(X, T, U, V, U_{X X}, U_{X X X}, \ldots\right) .
\end{gathered}
$$

Suppose also that a one-parameter Lie group of transformations is given as follows:

$$
\begin{aligned}
& \bar{X}=X+\epsilon \xi_{1}(X, T, U, V)+O\left(\epsilon^{2}\right), \\
& \bar{T}=T+\epsilon \xi_{2}(X, T, U, V)+O\left(\epsilon^{2}\right), \\
& \bar{U}=U+\epsilon \eta_{1}(X, T, U, V)+O\left(\epsilon^{2}\right), \\
& \bar{V}=V+\epsilon \eta_{2}(X, T, U, V)+O\left(\epsilon^{2}\right), \\
& \frac{\partial^{\alpha} \bar{U}}{\partial \bar{T}^{\alpha}}=\frac{\partial^{\alpha} U}{\partial T^{\alpha}}+\epsilon \eta_{1}^{\alpha, T}(X, T, U, V)+O\left(\epsilon^{2}\right), \\
& \frac{\partial^{\alpha} \bar{v}}{\partial \bar{t}^{\alpha}}=\frac{\partial^{\alpha} v}{\partial t^{\alpha}}+\epsilon \eta_{2}^{\alpha, t}(X, T, U, V)+O\left(\epsilon^{2}\right), \\
& \frac{\partial \bar{U}}{\partial \bar{X}}=\frac{\partial U}{\partial X}+\epsilon \eta_{1}^{X}(X, T, U, V)+O\left(\epsilon^{2}\right), \\
& \frac{\partial \bar{V}}{\partial \bar{X}}=\frac{\partial V}{\partial X}+\epsilon \eta_{2}^{X}(X, T, U, V)+O\left(\epsilon^{2}\right), \\
& \frac{\partial^{2} \bar{U}}{\partial \bar{X}^{2}}=\frac{\partial^{2} U}{\partial X^{2}}+\epsilon \eta_{1}^{X X}(X, T, U, V)+O\left(\epsilon^{2}\right), \\
& \frac{\partial^{2} \bar{V}}{\partial \bar{X}^{2}}=\frac{\partial^{2} V}{\partial X^{2}}+\epsilon \eta_{2}^{X X}(X, T, U, V)+O\left(\epsilon^{2}\right), \\
& \frac{\partial^{3} \bar{U}}{\partial \bar{X}^{3}}=\frac{\partial^{3} U}{\partial X^{3}}+\epsilon \eta_{1}^{X X X}(X, T, U, V)+O\left(\epsilon^{2}\right), \\
& \frac{\partial^{3} \bar{V}}{\partial \bar{X}^{3}}=\frac{\partial^{3} U}{\partial X^{3}}+\epsilon \eta_{2}^{X X X}(X, T, U, V)+O\left(\epsilon^{2}\right),
\end{aligned}
$$

where $\xi_{1}, \xi_{2}, \eta_{1}, \eta_{2}$ are the infinitesimal operators, $\eta_{1}^{\alpha, t}, \eta_{2}^{\alpha, t}$ are the extended infinitesimals of order $\alpha$, and $\eta_{1}^{X}, \eta_{2}^{X}, \eta_{1}^{X X}, \eta_{2}^{X X}, \eta_{1}^{X X X}, \eta_{1}^{X X X}$ are the integer order extended infinitesimals. Surmise that we have vector fields as follows:

$$
\mathbf{X}=\xi_{1} \frac{\partial}{\partial X}+\xi_{2} \frac{\partial}{\partial T}+\eta_{1} \frac{\partial}{\partial U}+\eta_{2} \frac{\partial}{\partial V}
$$


Equation (3) exhibits a point symmetry of Eq. (1) such that

$$
\left.P^{\alpha, i} r \mathbf{X}(\triangle)\right|_{\triangle=0}=0
$$

$i$ exhibits an order Eq. (1). Also, for the invariance condition, we have

$$
\left.\xi_{2}(X, T, U, V)\right|_{T=0}=0
$$

The $\alpha$ th extended infinitesimal related to the RL fractional time derivative with Eq. (7) is given as in $[38,40,41]$.

\section{The models}

The time fractional dispersive long-wave system is given by

$$
\begin{aligned}
& \frac{\partial^{\alpha} U}{\partial T^{\alpha}}=\left(U^{2}-U_{X}+2 V\right)_{X}, \\
& \frac{\partial^{\alpha} V}{\partial T^{\alpha}}=\left(2 U V+V_{X}\right)_{X},
\end{aligned}
$$

where $0<\alpha \leq 1$. If $\alpha=1$, Eq. (6) becomes

$$
\begin{aligned}
& \frac{\partial U}{\partial T}=\left(U^{2}-U_{X}+2 V\right)_{X}, \\
& \frac{\partial V}{\partial T}=\left(2 U V+V_{X}\right)_{X} .
\end{aligned}
$$

Equation (7) has well been known as DLWE [52]. In the concept of the spectral transform, Eq. (7) is analyzed in [52] and thereafter in [53], where it was related to the Schrödinger equation with linear spectral dependence in the potential. In hydrodynamics it exhibits the evolution of the horizontal velocity component of water waves propagating in both directions in an infinite narrow channel of constant depth and could be generated from the water wave equations by including one more order of nonlinearity than is done in deriving the Boussinesq equation. The integrability and derivation for Eq. (7) was presented in [54]. Furthermore, in [55] the authors related it directly to the spectral problem

$$
\left(\partial_{X}+\alpha+\beta \partial_{X}^{-1}\right) \phi=\lambda \phi
$$

Considering the invariance of Eq. (6) under the group of transformations Eq. (2), we get the following:

$$
\begin{aligned}
& \eta_{1}^{\alpha, T}-2 U \eta_{1}^{X}+2 \eta_{2}^{X}+\eta_{1}^{X X}=0, \\
& \eta_{1}^{\alpha, T}-2 U \eta_{1}^{X}+2 V \eta_{2}^{X}+\eta_{2}^{X X}=0 .
\end{aligned}
$$

Inserting the prolongations, we obtain determining equations. Solving the obtained determining equations, we acquire

$$
\xi_{1}=c_{3} \alpha X+c_{4}, \quad \xi_{2}=C_{1}+2 T c_{3}, \quad \eta_{1}=-U c_{3} \alpha, \quad \eta_{2}=-2 V c_{3} \alpha,
$$


and the corresponding Lie algebra is generated by the vector field below:

$$
X_{1}=\frac{\partial}{\partial X}, \quad X_{2}=\frac{\partial}{\partial T}, \quad X_{3}=\alpha X \frac{\partial}{\partial X}+2 T \frac{\partial}{\partial T}-U \alpha \frac{\partial}{\partial U}-2 V \alpha \frac{\partial}{\partial V} .
$$

The solutions of Eq. (11) lead to the following theorem.

Theorem 1 By using the similarity transformations $U=T^{-\frac{\alpha}{2}} f(\xi)$ and $V=T^{-\alpha} g(\xi)$ with the similarity variable $\xi=X T^{-\frac{\alpha}{2}}$, Eq. (6) attains to

$$
\begin{aligned}
& \left(P_{\frac{2}{\alpha}}^{1-\alpha-\frac{\alpha}{2}, \alpha} f\right)(\xi)-\left(f^{2}-f_{\xi}+2 g\right)_{\xi}=0 \\
& \left(P_{\frac{2}{\alpha}}^{1-2 \alpha, \alpha} g\right)(\xi)-\left(2 f g+g_{\xi}\right)_{\xi}=0 .
\end{aligned}
$$

Proof Similar steps can be found in [41].

\subsection{Conservation laws for Eq. (6)}

Several works involving the procedures for computing Cls of fractional PDEs were presented in numerous research works [56-60]. In this work, we apply the procedures presented in $[56,57]$ to establish Cls for Eq. (6). Surmise that the formal Lagrangian for Eq. (6) is given as

$$
\begin{aligned}
\mathcal{L}= & p(X, T)\left(\frac{\partial^{\alpha} U}{\partial T^{\alpha}}-2 U U_{X}+U_{X X}-2 V_{X}\right) \\
& +q(X, T)\left(\frac{\partial^{\alpha} V}{\partial T^{\alpha}}-2 V U_{X}-2 U V_{X}-V_{X X}\right) .
\end{aligned}
$$

The adjoint equations can be presented as follows:

$$
\begin{aligned}
& \frac{\delta \mathcal{L}}{\delta U}=F_{1}^{*}=\left(D_{T}^{\alpha}\right)^{*} p-2 u p_{X}-p_{X X}+2 V q_{x}=0 \\
& \frac{\delta \mathcal{L}}{\delta V}=F_{2}^{*}=\left(D_{T}^{\alpha}\right)^{*} q-2 U q_{X}-2 p_{X}+q_{X X}=0
\end{aligned}
$$

For Eq. (6) to be nonlinearly self-adjoint, Eq. (14) must hold for all solutions of Eq. (6) upon the following substitution:

$$
p=\Phi_{1}(X, T, U, V), \quad q=\Phi_{2}(X, T, U, V),
$$

such that $\Phi_{i} \neq 0$ for at least one $i(i=1,2)$. The conditions for the nonlinear self-adjointness can be presented as follows:

$$
\left.\frac{\delta \mathcal{L}}{\delta U}\right|_{\text {Eq. (15) }}=\lambda_{1}\left(\frac{\partial^{\alpha} U}{\partial T^{\alpha}}+\left(U^{2}-U_{X}+2 V\right)_{X}\right)+\lambda_{2}\left(\frac{\partial V}{\partial T}+\left(2 U V+V_{X}\right)_{X}\right)
$$

and

$$
\left.\frac{\delta \mathcal{L}}{\delta V}\right|_{\mathrm{Eq} \cdot(15)}=\lambda_{3}\left(\frac{\partial^{\alpha} U}{\partial T^{\alpha}}+\left(U^{2}-U_{X}+2 V\right)_{X}\right)+\lambda_{4}\left(\frac{\partial V}{\partial T}+\left(2 U V+V_{X}\right)_{X}\right) .
$$


Hence, applying Eq. (15) along with its associated derivatives in Eqs. (16) and (17) thereby solving the resulting expressions, the following solution is obtained:

$$
\begin{aligned}
& \lambda_{1}=\lambda_{2}=\lambda_{3}=\lambda_{4}=0, \\
& \Phi_{1}(X, T, U, V)=0, \quad \Phi_{2}(X, T, U, V)=A_{2},
\end{aligned}
$$

where $A_{2}$ is an arbitrary constant. Thus, Eq. (15) is nonlinearly self-adjoint.

The characteristic functions are given as follows:

$$
\begin{aligned}
& W_{1}=-U \alpha-2 T U_{T}-\alpha X U_{X}, \quad W_{2}=-V \alpha-2 T V_{T}-\alpha X V_{X}, \\
& W_{3}=-U_{X}, \quad W_{4}=-V_{X} W_{5}=-U_{T}, \quad W_{5}=-V_{T} .
\end{aligned}
$$

Using Eq. (19) and setting $A_{2}=1$, the conserved vectors are:

The $X$-components $C_{i}^{X}$ associated with Eq. (19) are as follows:

$$
\begin{aligned}
C_{1}^{X}= & 2(U+V)\left(-U \alpha-2 T U_{T}-\alpha X U_{X}-V \alpha-2 T V_{T}-\alpha X V_{X}\right) \\
& -\left(\left(U_{X}+V_{X}\right)+\alpha X\left(U_{X X}+V_{X X}\right)+2 T\left(U_{X T}+V_{X T}\right)\right), \\
C_{2}^{X}= & 2(U+V)\left(U_{X}+U V_{X}\right)+U_{X X}+V_{X X}, \\
C_{3}^{X}= & 2(U+V)\left(U_{T}+U V_{T}\right)+U_{X X}+V_{X X} .
\end{aligned}
$$

The $t$-components $C_{i}^{t}$ are given as follows:

Case 1. When $\alpha \in(0,1)$, the conserved vectors are

$$
\begin{aligned}
C_{1}^{T}= & -\alpha\left(I_{T}^{1-\alpha}(U)+I_{T}^{1-\alpha}(V)\right) \\
& -\alpha X\left(I_{T}^{1-\alpha}\left(U_{X}\right)+I_{T}^{1-\alpha}\left(V_{X}\right)\right)-2\left(I_{T}^{1-\alpha}\left(T U_{T}\right)+I_{T}^{1-\alpha}\left(T V_{T}\right)\right), \\
C_{2}^{T}= & -I_{T}^{1-\alpha}\left(V_{X}\right) .
\end{aligned}
$$

Case 2. When $\alpha \in(1,2)$, the conserved vectors are

$$
\begin{aligned}
C_{1}^{T}= & -\alpha\left(D_{T}^{1-\alpha}(U)+D_{T}^{1-\alpha}(V)\right) \\
& -\alpha X\left(D_{T}^{1-\alpha}\left(U_{X}\right)+D_{T}^{1-\alpha}\left(V_{X}\right)\right)-2\left(D_{T}^{1-\alpha}\left(T U_{T}\right)+D_{T}^{1-\alpha}\left(T V_{T}\right)\right), \\
C_{2}^{T}= & -D_{T}^{1-\alpha}\left(V_{X}\right) .
\end{aligned}
$$

\section{Soliton-like solutions}

In this section, by means of the conformable derivative [11, 12] and the GPR method [61], some soliton-like solutions will be presented for Eq. (6). Applying the conformable derivative and plugging the transformation $U(X, T))=U(\eta), V(X, T)=V(\eta), \eta=k\left(X-\Lambda \frac{T^{\alpha}}{\alpha}\right)$ in Eq. (6) yields

$$
\begin{aligned}
& k^{2} U^{\prime \prime}-k \Lambda U^{\prime}-2 k U U^{\prime}-2 k V^{\prime}=0, \\
& k^{2} V^{\prime \prime}+2 k V U^{\prime}+2 k U V^{\prime}+k \Lambda V^{\prime}=0 .
\end{aligned}
$$


According to the GPR method [61], applying homogeneous principles in Eq. (20), we can have the following solutions:

$$
\begin{aligned}
& U(\eta)=A_{0}+A_{1} \sigma(\eta)+B_{1} \tau(\eta), \\
& V(\eta)=F_{0}+F_{1} \sigma(\eta)+G_{1} \tau(\eta),
\end{aligned}
$$

where $A_{0}, A_{1}, B_{1}, F_{0}, F_{1}, G_{1}$ are constants and will be found later. The functions $\sigma(\eta)$ and $\tau(\eta)$ satisfy the ODE

$$
\begin{aligned}
& \sigma^{\prime}(\eta)=\epsilon \sigma(\eta) \tau(\eta), \\
& \tau^{\prime}(\eta)=R+\epsilon \tau^{2}(\eta)-\mu \sigma(\eta), \quad \epsilon= \pm 1,
\end{aligned}
$$

where

$$
\tau^{2}(\eta)=-\epsilon\left(R-2 \mu \sigma(\eta)+\frac{\mu^{2}-1}{R} \sigma^{2}(\eta)\right),
$$

where $R$ and $\mu$ are nonzero constants. Plugging Eq. (21) along with Eqs. (22) and (23) into Eq. (20), we obtain large algebraic expressions. Collecting terms in $(\sigma(\eta))^{3},(\sigma(\eta))^{2}$, $(\sigma(\eta))^{1},(\sigma(\eta))^{0},(\sigma(\eta))^{2} \tau(\eta),(\sigma(\eta)) \tau(\eta), \tau(\eta)$ from the obtained algebraic expressions gives systems of algebraic expressions. Solving the obtained systems yields the following.

Results: $\epsilon=-1, A_{0}=A_{0}, \Lambda=-2 A_{0}, A_{1}=0, B_{1}=B_{1}, F_{0}=0, F_{1}=\frac{1}{2} B_{1} \mu\left(2 B_{1}+k\right), G_{1}=0$, $\mu R \neq 0$. These results give the following soliton-like solutions: For $\epsilon=-1, R \neq 0$, we acquire the soliton-like solution

$$
\begin{aligned}
U(X, T) & =A_{0}+B_{1} \frac{R \tanh (\sqrt{R} \eta)}{\mu \operatorname{sech}(\sqrt{R} \eta)+1}, \\
V(X, T) & =\frac{1}{2} B_{1} \mu\left(2 B_{1}+k\right) \frac{R \operatorname{sech}(\sqrt{R} \eta)}{\mu \operatorname{sech}(\sqrt{R} \eta)+1},
\end{aligned}
$$

and

$$
\begin{aligned}
& U(X, T)=A_{0}+B_{1} \frac{R \operatorname{coth}(\sqrt{R} \eta)}{\mu \operatorname{csch}(\sqrt{R} \eta)+1}, \\
& V(X, T)=\frac{1}{2} B_{1} \mu\left(2 B_{1}+k\right) \frac{R \operatorname{csch}(\sqrt{R} \eta)}{\mu \operatorname{csch}(\sqrt{R} \eta)+1},
\end{aligned}
$$

where $\eta=k\left(X-\Lambda \frac{T^{\alpha}}{\alpha}\right)$. Some physical features of the obtained soliton-like are illustrated in Figs. 1 and 2.

\section{Conservation laws for Eq. (7) by multiplier}

The description for Cls via the multiplier technique was presented in [62]. Here, we apply the same process to obtain Cls for the integer order of DLWE reported in Eq. (7) by using a first order multiplier that is $\Lambda^{1}\left(X, T, U, V, U_{X}, V_{X}, U_{T}, V_{T}\right), \Lambda^{2}\left(X, T, U, V, U_{X}, V_{X}, U_{T}, V_{T}\right)$. We obtain the first order multiplier for Eq. (7) given by

$$
\begin{aligned}
& \Lambda^{1}=u c_{2}, \\
& \Lambda^{2}=-2 c_{1} U V+c_{2} U^{2}-c_{1} V_{X}-c_{2} U_{X}+2 c_{2} V,
\end{aligned}
$$




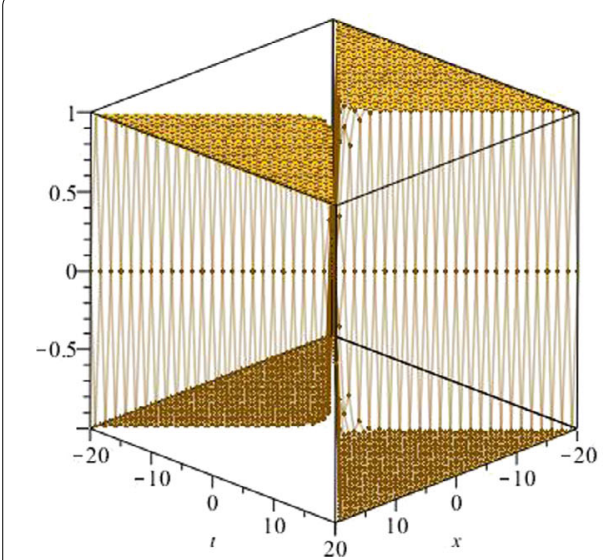

(a)

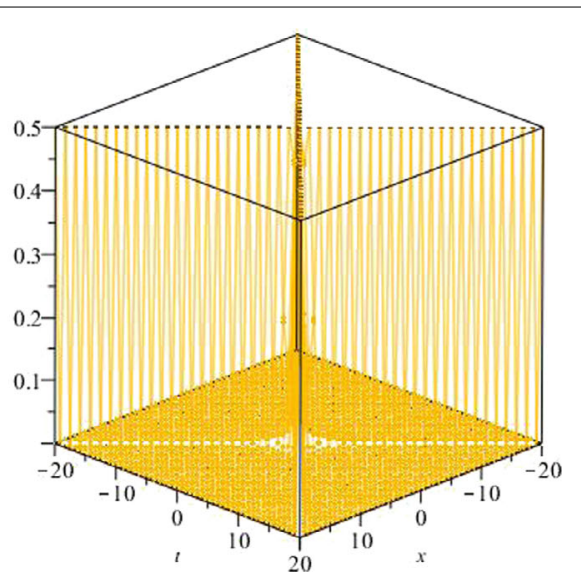

(b)

Figure $13 \mathrm{D}$ plot of (25), with $\alpha=0.75, \mu=1.5, k=2, A_{0}=B_{1}=1$, respectively

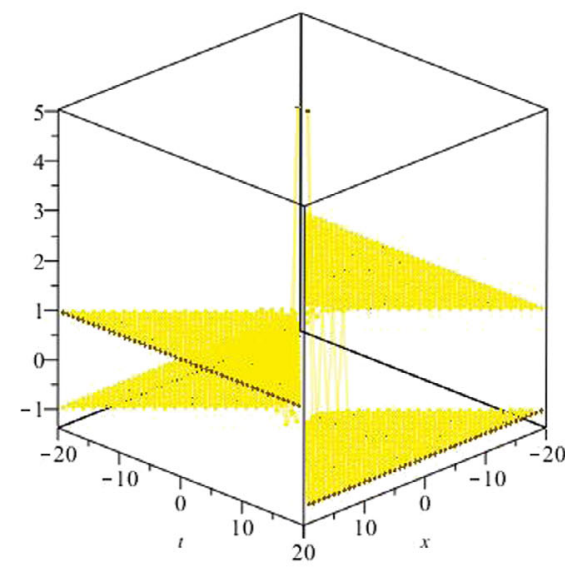

(a)

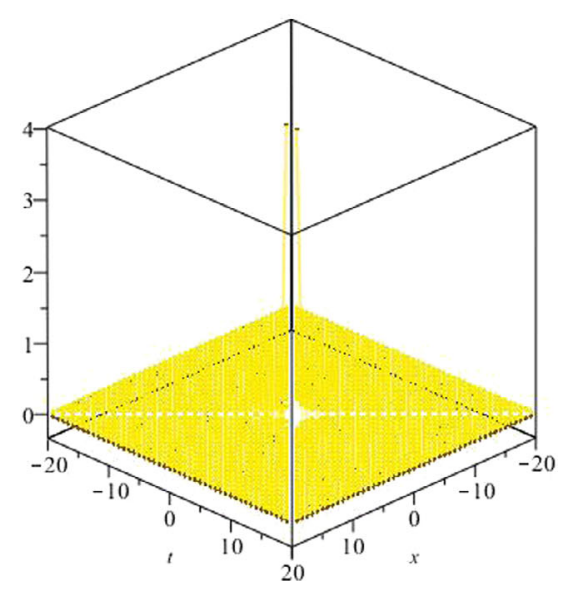

(b)

Figure $23 \mathrm{D}$ plot of (26), with $\alpha=0.75, \mu=1.5, k=2, A_{0}=B_{1}=1$, respectively

where $c_{1}, c_{2}$ are arbitrary constants. Therefore, the multipliers for the non trivial local Cls involving the cases isolated by free constants can be obtained as follows:

1.

$$
\begin{aligned}
& \Lambda^{1}=0, \\
& \Lambda^{2}=1,
\end{aligned}
$$

which gives the following fluxes:

$$
\begin{aligned}
& \mathbf{C}^{T}=0, \\
& \mathbf{C}^{X}=-2 U V-V_{X} .
\end{aligned}
$$


2.

$$
\begin{aligned}
& \Lambda^{1}=1, \\
& \Lambda^{2}=0,
\end{aligned}
$$

which yields the following fluxes:

$$
\begin{aligned}
& \mathbf{C}^{T}=U, \\
& \mathbf{C}^{X}=U^{2}-U_{X}+2 V .
\end{aligned}
$$

\subsection{Stability analysis to Eq. (7)}

In this subsection, the concept of linear stability analysis [63-65] will be applied to investigate the stability analysis for the governing equation. Consider the integer order for DLWE as in Eq. (7). Then, by considering the perturbed solution of the form

$$
\begin{aligned}
& U(X, T)=P_{1}+\epsilon w(X, T), \\
& V(X, T)=P_{2}+\tau r(X, T),
\end{aligned}
$$

it is easy to see that any constants $P_{1}$ and $P_{2}$ are a steady state solution of Eq. (7). Plugging Eq. (32) to Eq. (7), we obtain

$$
\begin{aligned}
& -\epsilon w_{T}+2 \tau r_{X}+2 \epsilon P_{1} w_{X}+2 \epsilon^{2} w w_{X}-\epsilon w_{X X}=0, \\
& -\tau r_{T}+2 \tau P_{1} r_{X}+2 \epsilon \tau w r_{X}+2 \epsilon \tau r w_{X}+2 \epsilon P_{2} w_{X}+\epsilon w_{X X}=0 .
\end{aligned}
$$

Linearizing (33) in $\epsilon$ and $\tau$ gives

$$
\begin{aligned}
& k^{2} \epsilon \alpha_{1}-i \epsilon \omega \alpha_{1}+2 i k \epsilon P_{1} \alpha_{1}+2 i k \tau \alpha_{2}=0, \\
& k^{2} \epsilon \alpha_{1}-2 i k \epsilon P_{2} \alpha_{1}+i \tau \omega \alpha_{2}-2 i k \tau P_{1} \alpha_{2}=0 .
\end{aligned}
$$

Surmise that Eq. (34) has solutions given by

$$
\begin{aligned}
& w(X, T)=\alpha_{1} e^{(i k X+i \omega T)}, \\
& r(X, T)=\alpha_{2} e^{(i k X+i \omega T)},
\end{aligned}
$$

$k$ denotes a normalized wave number. Plugging Eq. (35) into Eq. (34) yields

$$
\begin{aligned}
& i k^{2} \epsilon \beta \alpha_{1}+\epsilon \omega \alpha_{1}+k \epsilon P_{1} \alpha_{1}+k \tau \alpha_{2}=0 \\
& -i k^{3} \alpha \epsilon \alpha_{1}+i k \epsilon P_{2} \alpha_{1}+k^{2} \beta \tau \alpha_{2}+i \tau \omega \alpha_{2}+i k \tau P_{1} \alpha_{2}=0
\end{aligned}
$$

Collecting terms with $\alpha_{1}, \alpha_{2}$ gives

$$
\left(\begin{array}{cc}
\epsilon\left(k^{2}-i \omega+2 i k P_{1}\right) & 2 i k \tau \\
k \epsilon\left(k-2 i P_{2}\right) & i \tau\left(\omega-2 k P_{1}\right)
\end{array}\right)\left(\begin{array}{l}
\alpha_{1} \\
\alpha_{2}
\end{array}\right)=\left(\begin{array}{l}
0 \\
0
\end{array}\right)
$$




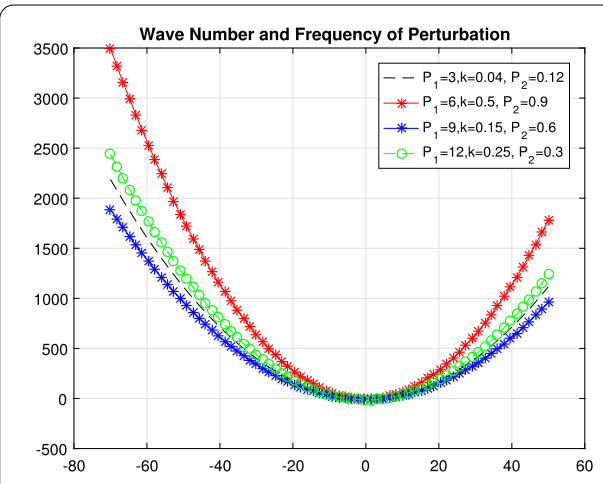

(a)

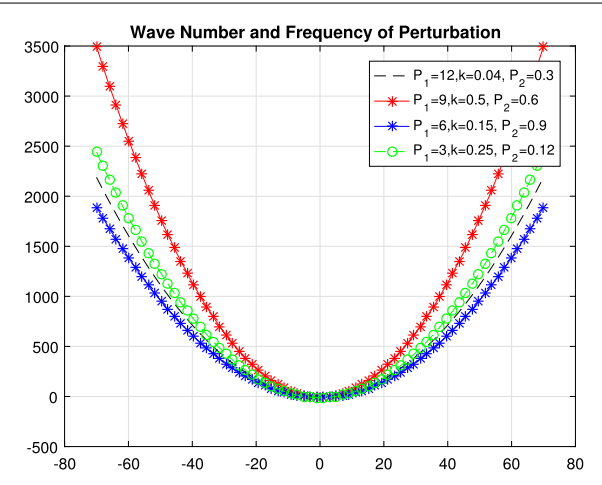

(b)

Figure 3 Frequency of the perturbation against the wave number with different parameter values

and taking the determinant of the above yields

$$
-2 i \alpha_{1} k^{3} \tau \epsilon-2 i k^{3} P_{1} \tau \epsilon-4 \alpha_{1} k^{2} P_{2} \tau \epsilon+4 k^{2} P_{1}^{2} \tau \epsilon+i k^{2} \tau \omega \epsilon-4 k P_{1} \tau \omega \epsilon+\tau \omega^{2} \epsilon=0 .
$$

Solving for $\omega$ yields

$$
\omega(k)=\frac{1}{2}\left(4 k P_{1}-i\left(k^{2}-\sqrt{k^{4}+8 i k^{3}-16 k^{2} P_{2}}\right)\right) .
$$

The relations for the dispersion in Eq. (39) will be investigated. The sign of the real part (Re) of $\omega$ suggests that either the solution will become bigger or vanish in a given period of time. When the sign of Re for $\omega(k)$ is negative for all $k$, thus any superposition of $e^{(i \omega t+i k x)}$ will come to vanished. Moreover, if the Re is positive for some $k$, then with time some components of a superposition will become much bigger. The former case is said to be stable, otherwise unstable. If the maximum of the Re is exactly zero, then it is said to be marginally stable. It is more difficult to assess the long term behavior in this case. Thus, from Eq. (39) one can observe that the Re is always negative for $k<0, P_{1}>0$ and $k>0$, $P_{1}<0$, which implies that the dispersion relation is stable. If $k<0, P_{1}<0$ and $k>0, P_{1}>0$, the Re will be positive, hence in this case the dispersion is unstable. When $k=0$, the $\operatorname{Re}$ will be zero, which suggests that the dispersion is marginally stable in this case. In order to see the mechanism of Eq. (39), we plot Fig. 3.

\section{Conclusion}

We investigated time fractional DLWE and its corresponding integer order. The symmetry properties and reductions were derived. We constructed the Cls with RL for the time fractional DLWE via new conservation theorem. The conformable derivative was employed to establish soliton-like solutions for the time fractional DLWE by using the generalized projective method (GPM). Moreover, the Cls via the multiplier technique and the stability analysis via the concept of linear stability analysis for the integer order DLWE were established. Some graphical features for the obtained results were also presented. 


\section{Competing interests}

The authors declare that they have no competing interests.

\section{Authors' contributions}

All authors read and approved the final version of the manuscript.

\section{Author details}

${ }^{1}$ Department of Mathematics, Science Faculty, Firat University, Elazig, Turkey. ${ }^{2}$ Department of Mathematics, Science Faculty, Federal University Dutse, Jigawa, Nigeria. ${ }^{3}$ Department of Mathematics, Cankaya University, Ankara, Turkey. ${ }^{4}$ Institute of Space Sciences, Bucharest, Romania.

\section{Publisher's Note}

Springer Nature remains neutral with regard to jurisdictional claims in published maps and institutional affiliations.

Received: 25 July 2018 Accepted: 23 August 2018 Published online: 12 September 2018

\section{References}

1. Caputo, M., Mainardi, F.: A new dissipation model based on memory mechanism. Pure Appl. Geophys. 91, 134-147 (1971)

2. Caputo, M., Fabrizio, M.: A new definition of fractional derivative without singular kernel. Prog. Fract. Differ. Appl. 1(2), 73-85 (2015)

3. Atangana, A., Baleanu, D.: New fractional derivatives with nonlocal and non-singular kernel: theory and application to heat transfer model. Therm. Sci. 20(2), 763-769 (2016)

4. Algahtani, O.J.J.: Comparing the Atangana-Baleanu and Caputo-Fabrizio derivative with fractional order for Allen Cahn model. Chaos Solitons Fractals 89, 552-559 (2016)

5. Podlubny, I.: Fractional Differential Equations. Academic Press, San Diego (2006)

6. Diethelm, K.: The Analysis of Fractional Differential Equations. Springer, New York (2010)

7. Machado, J.T., Kiryakova, V., Mainardi, F.: Recent History of Fractional Calculus. Longman, London (2010)

8. Samko, S.G., Kilbas, A.A., Marichev, O.L.: Fractional Integrals and Derivatives: Theory and Applications. Gordon \& Breach, New York (1993)

9. Miller, K.S., Ross, B.: An Introduction to the Fractional Calculus and Fractional Differential Equations. Wiley, New York (1993)

10. Atangana, A., Baleanu, D., Alsaedi, A.: Analysis of time-fractional Hunter-Saxton equation: a model of neumatic liquid crystal. Open Phys. 14, 145-149 (2016)

11. Khalil, R., Al Horani, M., Yousef, A., Sababheh, M.: A new definition of fractional derivative. J. Comput. Appl. Math. 264, 65-70 (2014)

12. Atangana, A., Baleanu, D., Alsaedi, A.: New properties of conformable derivative. Open Math. 13, $889-898$ (2015)

13. Zheng, A., Feng, Y., Wang, W.: The Hyers-Ulam stability of the conformable fractional differential equation. Math. Æterna 5, 485-492 (2015)

14. Iyiola, O.S., Nwaeze, E.R.: Some new results on the new conformable fractional calculus with application using D'Alambert approach. Prog. Fract. Differ. Appl. 2, 115-122 (2016)

15. Michal, P., Skripkov, L.P.: Sturm's theorems for conformable fractional differential equations. Math. Commun. 21, 273-281 (2016)

16. Fuat, U., Mehmet, Z.S.: Explicit bounds on certain integral inequalities via conformable fractional calculus. Cogent Math. 4, Article ID 1277505 (2017)

17. Bluman, G.W., Cheviakov, A.F., Anco, S.C.: Applications of Symmetry Methods to Partial Differential Equations. Springer, New York (2010)

18. Leveque, R.J.: Numerical Methods for Conservation Laws. Birkhäuser Verlag, Basel (1992)

19. Naz, R.: Conservation laws for some systems of nonlinear partial differential equations via multiplier approach. J. Appl. Math. 2012, Article ID 871253 (2012)

20. Noether, E.: Invariant variation problems. Nachr. Akad. Wiss. Gött. Math.-Phys. Kl. 2, 235-257 (1918)

21. Agrawal, O.P.: Formulation of Euler-Lagrange equations for fractional variational problems. J. Math. Appl. 272, 368-379 (2002)

22. Frederico, G.S.F., Torres, D.F.M.: A formulation of Noether's theorem for fractional problems of the calculus of variations. J. Math. Anal. 334, 834-846 (2007)

23. Baleanu, D.: About fractional quantization and fractional variational principles. Commun. Nonlinear Sci. Numer. Simul. $14,2520-2523$ (2009)

24. Yang, X.J., Gao, F., Srivastava, H.M.: New rheological models within local fractional derivative. Rom. Rep. Phys. 69 Article ID 113 (2017)

25. Eslami, M., Vajargah, B.F., Mirzazadeh, M., Biswas, A.: Application of first integral method to fractional partial differential equations. Indian J. Phys. 88(2), 177-184 (2014)

26. Mirzazadeh, M.: Analytical study of solitons to nonlinear time fractional parabolic equations. Nonlinear Dyn. 85(4), 2569-2576 (2016)

27. Biswas, A., Mirzazadeh, M., Eslami, M., Milovic, D., Belic, M.: Solitons in optical metamaterials by functional variable method and first integral approach. Frequenz 68(11-12), 525-530 (2014)

28. Eslami, M., Mirzazadeh, M.: Optical solitons with Biswas-Milovic equation for power law and dual-power law nonlinearities. Nonlinear Dyn. 83(1-2), 731-738 (2016)

29. Mirzazadeh, M., Eslami, M., Zerrad, E., Mahmood, M.F., Biswas, A., Belic, M.: Optical solitons in nonlinear directional couplers by sine-cosine function method and Bernoulli's equation approach. Nonlinear Dyn. 81(4), 1933-1949 (2015)

30. Arnous, A.H., Ullah, M.Z., Moshokoa, S.P., Zhou, Q., Triki, H., Mirzazadeh, M., Biswas, A.: Optical solitons in nonlinear directional couplers with trial function scheme. Nonlinear Dyn. 88(3), 1891-1915 (2017) 
31. Eslami, M., Mirzazadeh, M.: First integral method to look for exact solutions of a variety of Boussinesq-like equations. Ocean Eng. 83, 133-137 (2014)

32. Mirzazadeh, M., Yıldıım, Y., Yaşar, E., Triki, H., Zhou, Q., Moshokoa, S.P., Ullah, M.Z., Seadawy, A.R., Biswas, A., Belic, M.: Optical solitons and conservation law of Kundu-Eckhaus equation. Optik 154, 551-557 (2018)

33. Qin, Z., Mirzazadeh, M., Ekici, M., Sonmezoglu, A.: Analytical study of solitons in non-Kerr nonlinear negative-index materials. Nonlinear Dyn. 86(1), 623-638 (2016)

34. Qin, Z., Ekici, M., Sonmezoglu, A., Mirzazadeh, M., Eslami, M.: Optical solitons with Biswas-Milovic equation by extended trial equation method. Nonlinear Dyn. 84(4), 1883-1900 (2016)

35. Mirzazadeh, M., Ekici, M., Sonmezoglu, A., Eslami, M., Qin, Z., Kara, A.H., Milovic, D., Fayequa, B.M., Biswas, A., Belic, M.: Optical solitons with complex Ginzburg-Landau equation. Nonlinear Dyn. 85(3), 1979-2016 (2016)

36. Ashrafi, S., Golmankhaneh, A.K., Baleanu, D.: Generalized master equation, Bohr's model, and multipoles on fractals. Rom. Rep. Phys. 69, Article ID 117 (2017)

37. Yang, X.J.: New general fractional-order rheological models with kernels of Mittag-Leffler functions. Rom. Rep. Phys. 69, Article ID 118 (2017)

38. Hashemi, M.S.: Group analysis and exact solutions of the time fractional Fokker-Planck equation. Physica A 417, $141-149(2015)$

39. Gazizov, R.K., Ibragimov, N.H., Lukashchuk, S.Y.: Nonlinear self-adjointness, conservation laws and exact solutions of time-fractional Kompaneets equations. Commun. Nonlinear Sci. Numer. Simul. 23, 153-163 (2014)

40. Lukashchuk, S.Y.: Conservation laws for time-fractional subdiffusion and diffusion-wave equations. Nonlinear Dyn. 80, 791-802 (2015)

41. Baleanu, D., Inc, M., Yusuf, A., Aliyu, A.I.: Time fractional third-order evolution equation: symmetry analysis, explicit solutions, and conservation laws. J. Comput. Nonlinear Dyn. 13, Article ID 021011 (2018)

42. Baleanu, D., Inc, M., Yusuf, A., Aliyu, A.I.: Lie symmetry analysis, exact solutions and conservation laws for the time fractional modified Zakharov-Kuznetsov equation. Nonlinear Anal., Model. Control 22, 861-876 (2017)

43. Inc, M., Yusuf, A., Aliyu, A.I., Baleanu, D.: Time-fractional Cahn-Allen and time-fractional Klein-Gordon equations: Lie symmetry analysis, explicit solutions and convergence analysis. Physica A 493, 94-106 (2018)

44. Baleanu, D., Inc, M., Yusuf, A., Aliyu, A.I.: Lie symmetry analysis, exact solutions and conservation laws for the time fractional Caudrey-Dodd-Gibbon-Sawada-Kotera equation. Commun. Nonlinear Sci. Numer. Simul. 59, 222-234 (2018)

45. Hadi, R., Hira, T., Mostafa, E., Mirzazadeh, M., Qin, Z:: New exact solutions of nonlinear conformable time-fractional Phi-4 equation. Chin. J. Phys. (2018). https://doi.org/10.1016/j.cjph.2018.08.001

46. Yujia, Z., Chunyu, Y., Weitian, Y., Mirzazadeh, M., Qin, Z., Wenjun, L.: Interactions of vector anti-dark solitons for the coupled nonlinear Schrodinger equation in inhomogeneous fibers. Nonlinear Dyn. (2018). https://doi.org/10.1007/s11071-018-4428-2

47. Xiaoyan, L., Houria, T., Qin, Z., Wenjun, L., Biswas, A.: Analytic study on interactions between periodic solitons with controllable parameters. Nonlinear Dyn. (2018). https://doi.org/10.1007/s11071-018-4387-7

48. Asad, Z., Nauman, R., Mirzazadeh, M., Wenjun, L., Qin, Z.: Analytic study on optical solitons in parity-time-symmetric mixed linear and nonlinear modulation lattices with non-Kerr nonlinearities. Optik (2018). https://doi.org/10.1016/j.ijleo.2018.08.023

49. Osman, M.S., Alper, K., Hadi, R., Mirzazadeh, M., Eslami, M., Qin, Z.: The unified method for conformable time fractional Schrodinger equation with perturbation terms. Chin. J. Phys. (2018). https://doi.org/10.1016/j.cjph.2018.06.009

50. Guo, H., Zhang, X., Ma, G., Zhang, X., Yang, C., Zhou, Q., Liu, W.: Analytic study on interactions of some types of solitary waves. Optik 164, 132-137 (2018)

51. Yu, W., Ekici, M., Mirzazadeh, M., Zhou, Q., Liu, W.: Periodic oscillations of dark solitons in nonlinear optics. Optik 165 341-344 (2018)

52. Kaup, D.J.: A higher-order water-wave equation and the method for solving it. Prog. Theor. Phys. 54, 396-408 (1975)

53. Matveev, V.B., Yavor, M.I.: Solutions presque periodiques et a Nsolitons de l'equation hydrodynamic non lineaire de Kaup. Ann. Inst. Henri Poincaré 31, 25-41 (1979)

54. Boiti, M., Leon, J.P., Pempinelli, F.: Integrable two-dimensional generalisation of the sine and sinh-Gordon equations. Inverse Probl. 3, 37-49 (1987)

55. Kupershmidt, B.A.: Mathematics of dispersive water waves. Commun. Math. Phys. 99, 51-73 (2015)

56. Singla, K., Gupta, R.K.: On invariant analysis of some time fractional nonlinear systems of partial differential equations. II. J. Math. Phys. 57, Article ID 101504 (2016)

57. Singla, K., Gupta, R.K.: On invariant analysis of space-time fractional nonlinear systems of partial differential equations. II. J. Math. Phys. 58, Article ID 051503 (2017)

58. Majlesi, A., Ghehsareha, H.R., Zaghian, A.: On the fractional Jaulent-Miodek equation associated with energy-dependent Schrodinger potential: Lie symmetry reductions, explicit exact solutions and conservation laws. Eur. Phys. J. Plus 132, Article ID 516 (2017)

59. Ibragimov, N.H., Avdonin, E.D.: Nonlinear selfadjointness, conservation laws, and the construction of solutions of partial differential equations using conservation laws. Russ. Math. Surv. 68, 889-921 (2013)

60. Baleanu, D., Inc, M., Yusuf, A., Aliyu, A.I.: Space-time fractional Rosenou-Haynam equation: Lie symmetry analysis, explicit solutions and conservation laws. Adv. Differ. Equ. 2018, Article ID 46 (2018)

61. Li, B., Chen, Y:: Nonlinear partial differential equations solved by projective Riccati equations ansatz. Z. Naturforsch. $58,511-519$ (2003)

62. Buhe, E., Bluman, G.W.: Symmetry reductions, exact solutions, and conservation laws of the generalized Zakharov equations. J. Math. Phys. 56, Article ID 101501 (2015)

63. Agrawal, G.P.: Nonlinear Fiber Optics, 5th edn. Elsevier, New York (2013)

64. Saha, M., Sarma, A.K.: Solitary wave solutions and modulation instability analysis of the nonlinear Schrodinger equation with higher order dispersion and nonlinear terms. Commun. Nonlinear Sci. Numer. Simul. 18, 2420-2425 (2013)

65. Inc, M., Yusuf, A., Aliyu, A.I., Baleanu, D.: Soliton solutions and stability analysis for some conformable nonlinear partial differential equations in mathematical physics. Opt. Quantum Electron. 50, Article ID 190 (2018) 